\title{
TRABAJO EN EQUIPO Y CALIDAD DE LA ATENCIÓN EN SALUD
}

\author{
TEAMWORK AND HEALTH CARE QUALITY
}

\section{TRABALHO EM EQUIPE E QUALIDADE DOS CUIDADOS DE SAÚDE}

\author{
Tatiana Paravic Klijn* \\ María Elena Lagos Garrido**
}

\begin{abstract}
RESUMEN
Todas las organizaciones aspiran a alcanzar resultados funcionales e integrales para dar respuesta a las necesidades del medio interno y externo en el que se encuentran inmersas. La organización sanitaria no ha sido la excepción, por lo que proveer una atención con altos estándares de calidad se ha transformado en una prioridad a nivel mundial. En este sentido, se precisa poner en práctica una serie de elementos que confluyan funcionalmente para avanzar hacia la calidad de la atención en salud, en donde el trabajo en equipo, sus integrantes, el compromiso y la confianza entre las personas son claves para el logro de esta meta, especialmente para enfermería. El objetivo de este trabajo es describir la importancia del trabajo en equipo para la calidad de la atención en salud.
\end{abstract}

Palabras clave: Trabajo en equipo; Atención en salud; Atención de calidad; Gestión clínica.

\begin{abstract}
All organizations aspire to achieve functional and comprehensive results in order to meet the needs of the internal and external environment in which they are immersed. The healthcare organization has been no exception, and providing high quality care has become a worldwide priority. In this sense, it is necessary to put into practice a series of elements that functionally converge to move towards the quality of health care, where teamwork, team members, commitment and trust between people are keys to achieving this goal, especially for nursing. The objective of this work is to describe the importance of teamwork for the quality of health care.
\end{abstract}

Key words: Teamwork; Health care; Quality care; Clinical management.

\section{RESUMO}

Todas as organizaçóes aspiram a resultados funcionais e abrangentes para responder às necessidades do ambiente interno e externo em que estão inseridas. A organização de saúde não tem sido exceção, portanto, prestar

*Enfermera, Doctora en Enfermería, Departamento Fundamentos de Enfermería y Salud Pública, Facultad de Enfermería, Universidad de Concepción, Chile. ORCID: https://orcid.org/0000-0002-4034-0187 Email: tparavic@udec.cl

**Enfermera, Doctora en Enfermería, Departamento Fundamentos de Enfermería y Salud Pública, Facultad de Enfermería, Universidad de Concepción, Chile. ORCID: httos:orcid.org/0000-0002-7279-1193 Email: mariaelagos@udec.cl. Autora de correspondencia. 
cuidados com elevados padróes de qualidade tornou-se uma prioridade em todo o mundo. Nesse sentido, é necessário colocar em prática uma série de elementos que convergem funcionalmente para caminhar rumo à qualidade da assistência à saúde, onde o trabalho em equipe, seus integrantes, o compromisso e a confiança entre as pessoas são fundamentais para o alcance desse objetivo, especialmente para enfermagem. $\mathrm{O}$ objetivo deste documento é descrever a importância do trabalho de equipa para a qualidade dos cuidados de saúde.

Palavras-chave: Trabalho em equipe; Assistência médica; Atendimento de qualidade; Gestão clínica.

Fecha de recepción: 06/08/2021

Fecha de aceptación: 12/10/2021

\section{INTRODUCCIÓN}

Las organizaciones de salud tienen como objetivo promover la salud y el autocuidado, prevenir las enfermedades, mejorar los estilos de vida, y tratar las enfermedades con la mejor calidad posible, permitiendo cubrir las expectativas de los usuarios con la mayor satisfacción hacia el logro de los objetivos fijados conjuntamente.

En salud, el trabajo se desarrolla en un entorno de atención complejo, el cual está conformado por distintos elementos y actores que, interrelacionados, permiten avanzar hacia el logro de resultados funcionales, enfocados en la calidad y seguridad de la atención sanitaria ${ }^{(1-5)}$.

Las organizaciones de salud han visto que es necesario sincronizar las diversas actividades de sus usuarios internos en una conjunción, donde cada talento aporta su energía, interactúa y permite que fluya una dinámica colectiva, que lleve a la institución al cumplimiento de sus metas/objetivos con el mejor nivel de calidad posible. Es lo que llamamos trabajo en equipo, y cuyos resultados son el esfuerzo conjunto de todos sus integrantes. El compromiso, el sentido de responsabilidad y la confianza, entre otros, son la base para este trabajo en equipo, así como para la eficacia y calidad de los resultados ${ }^{(6-8)}$.

Es el trabajo en equipo la estrategia que mejor compatibiliza en la diversidad de procesos del que es objeto el usuario en la atención de salud y permite, desde distintas miradas, evaluar la calidad de lo que se entrega. Por lo anterior, el objetivo de este artículo es describir la importancia del trabajo en equipo para favorecer la calidad de la atención en salud.

Calidad de la atención en salud: La calidad de la atención se ha consignado como un concepto multidimensional que no ha tenido una única definición, ya que ha debido responder a la continua evolución de la atención sanitaria ${ }^{(1,}$ 2). Según el Instituto de Medicina de los Estados Unidos, "la calidad de la atención es el grado en que los servicios de salud, tanto para individuos y poblaciones, aumentan la probabilidad de obtener los resultados de salud deseados y son consistentes con el conocimiento profesional actual"(3). Por su parte, la Organización Mundial de la Salud (OMS) considera que la calidad de la atención se "centra en los sistemas de salud en su conjunto y en la calidad de los resultados que producen. Por esta razón, esta definición debe tomar una perspectiva de todo el sistema y reflejar una preocupación por los resultados logrados tanto a usuarios individuales del servicio como a comunidades enteras"(4). Sumado a ello, la OMS hace referencia a que la conceptualización de la calidad de la atención en salud considera atributos o dimensiones, tales como: la efectividad para lograr los objetivos propuestos para la atención basado en la evidencia; la eficiencia para lograr cuidados maximizando los recursos; la accesibilidad para proporcionar una atención de salud oportuna; la aceptabilidad centrada en el paciente, vinculada al grado de satisfacción usuaria, considerando las preferencias y expectativas de las personas y, por último, la equidad en la distribución de la atención y la seguridad, al disminuir el riesgo de daño asociado a la atención sanitaria ${ }^{(4)}$.

En Chile, el Observatorio de Calidad en Salud agrega tres elementos claves: continuidad, competencia profesional y oportunidad. Por continuidad señala que la atención se provea de forma ininterrumpida y coordinada, en y entre distintos proveedores e instituciones; respecto de la competencia profesional, hace referencia a la capacidad de los miembros del equipo de salud 
para solucionar problemas de salud y satisfacer las necesidades de sus usuarios, y la oportunidad hace referencia a que la entrega de servicios debe ser en el momento en que el usuario efectivamente lo requiera o necesite ${ }^{(5)}$.

Este conjunto de atributos conduce a la calidad de la atención en salud y deben ser vistos, no como elementos discretos, sino más bien como un continuo, en donde la suma de sus partes confluye funcionalmente para avanzar hacia la calidad de la atención en salud. En este sentido, el trabajo en equipo cobra una gran preponderancia para hacer frente a los desafíos conceptuales y prácticos de la calidad de la atención, porque es un proceso iterativo de constantes acuerdos y adaptaciones. Está basada en la evidencia y el equipo debe lograr consignar todas las acciones posibles para comprender todos sus atributos y sus interrelaciones y, de esta forma, lograr optimizar la salud de las personas ${ }^{(9)}$.

En la actualidad, se ha puesto al descubierto algunas dificultades relacionadas con el trabajo en equipo, la colaboración, el compromiso, la confianza, la comunicación, entre otras, las cuales continúan generando problemas en la calidad de la atención en salud y en la seguridad de los pacientes ${ }^{(10,11)}$. Así, se ha reportado que "las disciplinas profesionales se entrenan y trabajan por separado, y que necesitan de las habilidades para trabajar en equipo"(12). También la evidencia ha seńalado que mejorar el trabajo en equipo y optimizar el cómo las personas trabajan juntas, podría llevar a resultados más eficientes y eficaces. Lo anterior implica entender cómo cada miembro del equipo debe comunicarse y relacionarse, a la vez que comprender cómo la organización se adapta para lograr codiseñar y coproducir legítimas relaciones, basadas en un intercambio mutuo que impulse la adaptación de los equipos hacia el compromiso de una meta común, para el logro de una mejor calidad de la atención en salud ${ }^{(13-17)}$.

Trabajo en Equipo: El trabajo en equipo se ha transformado en una de las figuras más valiosas para lograr el éxito en cualquier proyecto que se quiera emprender. Es una forma de trabajo donde todos son responsables de las metas u objetivos de la organización, porque los esfuerzos de cada uno de los integrantes se potencian, aumentando la eficacia de sus resultados. Cada persona contribuye con sus opiniones y habilidades, armonizándolas para alcanzar los objetivos ${ }^{(18)}$.
Un equipo sería entonces dos o más individuos que interactúen socialmente (cara a cara o de forma virtual); poseen uno o más objetivos comunes y se han reunido para desarrollar una tarea organizacional relevante: demuestran interdependencia con respecto a la carga de trabajo, los objetivos y los resultados, tienen diferentes roles y responsabilidades y se encuentran inmersos en un sistema organizacional $^{(6)}$.

El trabajo en equipo permite el desarrollo de sus integrantes, comparten el liderazgo y disfrutan lo que están realizando; los resultados son el esfuerzo de $\operatorname{todos}^{(18)}$. "Cabe destacar que la implementación del trabajo en equipo supone una reducción de los niveles jerárquicos, en la medida en que las decisiones se van descentralizando hacia los niveles inferiores de la jerarquía. Esta descentralización es la esencia del proceso de autogestión de los equipos" ${ }^{\prime(19)}$. Se observa, además, que sus integrantes aumentan su compromiso, así como también el grado de placer y la calidad de los resultados de las tareas que emprenden ${ }^{(20)}$.

No siempre es fácil trabajar en equipo, porque lo conforman, generalmente, personas que tienen distintas historias de vida y experiencias, diversas culturas, distintas actitudes, diversos grados de compromiso para con la organización y distinta ética para realizar su trabajo. Por ello, cada persona aporta con sus acciones y su subjetividad, en beneficio de mejorar el trabajo y su entorno social ${ }^{21,}$ ${ }^{22)}$.

Para que el trabajo en equipo funcione debe haber compromiso con la organización y confianza entre los integrantes del equipo, así como también las organizaciones deben confiar en sus trabajadores, siendo este proceso bidireccional. Ese compromiso es el vínculo de lealtad por medio del cual el trabajador desea permanecer en la empresa debido a la motivación por el proyecto, el reconocimiento y/o la felicidad en el trabajo. El trabajador muestra entusiasmo por su trabajo y trabaja para el éxito de la empresa ${ }^{(23)}$. Así también el trabajador no buscará otras fuentes laborales, se sentirá atraído por la propia organización y ésta podrá retenerlo. Mientras más identificados e implicados en la organización en la que trabajan, mayores serán las probabilidades de que permanezcan en la misma ${ }^{(24)}$. El compromiso organizacional, entonces, es la decisión de continuar formando parte de ésta o dejarla. De esta manera, el concepto de compromiso organizacio- 
nal hace referencia a las actitudes del trabajador hacia el trabajo que realiza y a la identificación psicológica del mismo con la organización en la cual trabaja.

Contar con personal comprometido con la empresa repercute de manera positiva en el desempeño laboral del empleado, reduce los casos de absentismo, genera mayor satisfacción laboral, mejora el clima en la organización y reduce el índice de rotación ${ }^{(25)}$. Para contar con el compromiso de los integrantes de todo el equipo, es fundamental tenerse confianza y conocerse en profundidad. Cuanto más se conocen, más confianza hay en una relación y la confianza es una creencia que estima que una persona será capaz de actuar de una cierta manera frente a una determina situación ${ }^{(26,27)}$. La confianza es uno de los elementos más estimulantes en el desarrollo de las sociedades y en el crecimiento de las personas. Cuando hay confianza entre los integrantes de un equipo de trabajo, las personas se sienten seguras, sus compañeros son aliados y no rivales y saben lo que se espera de ellos y que entre todos deben avanzar. Son creativos, aprenden unos de otros, comparten sus conocimientos y asumen riesgos calculados. De esta forma cuando el nivel de confianza es elevado, trabajan en excelente forma y se preocupan los unos de los otros ${ }^{(27)}$.

Como se ha visto, el trabajo en equipo trae ventajas para las organizaciones sanitarias y también para los proveedores de salud, entre ellos los profesionales de enfermerías, quienes en Chile han asumido el rol de gestores del cuidado, velando por que los cuidados sean proporcionados con el más alto estándar de calidad. El profesional de enfermería, como líder del equipo de enfermería e integrante de un equipo multidisciplinario que otorga cuidados de salud, es un profesional que tiene un rol relevante en mantener la calidad de la atención de salud, donde la colaboración, el compromiso y la confianza juegan un rol en su máxima expresión ${ }^{(28-31)}$.

Para fortalecer el trabajo en equipo, es necesario potenciar una educación que promueva la construcción de saberes colaborativos y las formas de liderazgo integrador ${ }^{(17)}$, así como reforzar la confianza para apoyar la toma de decisiones compartidas, que permitan a los integrantes del equipo de salud comprometerse con una gestión organizacional que logre resultados de calidad ${ }^{(17}$, ${ }^{29,31)}$. Si este trabajo se desarrolla, además, en un ambiente armónico, se logra mayor bienestar y crecimiento como personas, grupo y sociedad.

\section{CONCLUSIÓN}

Para las personas que forman parte de una organización de salud, debe ser prioritario y estratégico trabajar en equipo, considerando sus elementos fundamentales, tales como: la confianza, compromiso, ejercicio de liderazgo compartido y descentralización jerárquica, donde el esfuerzo de todos es la responsabilidad conjunta en el logro de resultados con altos estándares de calidad de la atención en salud, y donde el profesional de enfermería, como integrante de un equipo multidisciplinario, actúa en uno de sus roles más relevantes, gestionando la calidad y seguridad en la atención de salud de los usuarios y sus familias.

\section{REFERENCIAS}

1. Donabedian A. Evaluating the quality of medical care. 1966. Milbank Q [Internet]. 2005 [citado 13 jul 2021]; 83(4): 691-729. Disponible en: https:// doi.org/10.1111/j.1468-0009.2005.00397.x

2. Ayanian JZ, Markel H. Donabedian's Lasting Framework for Health Care Quality N Engl J Med [Internet]. 2016 Jul [citado 13 jul 2021]; 375(3): 205-7. Disponible en: https://www.nejm.org/doi/ 10.1056/NEJMp1605101

3. Institute of Medicine (US). Committee to Design a Strategy for Quality Review and Assurance in Medicare; Lohr KN, editor. Medicare: A Strategy for Quality Assurance: VOLUME II Sources and Methods. Washington (DC): National Academies Press (US); 1990 [citado 13 jul 2021]. Disponible en: https://www.ncbi.nlm.nih.gov/books/NBK235 470/ doi: $10.17226 / 1548$

4. World Health Organization. Quality of Care: A process for making strategic choices in health systems [Internet]. Geneva 27, Switzerland: World Health Organization; 2006 [citado 14 jul 2021]. Disponible en: https://apps.who.int/iris/ bitstream/handle/10665/43470/9241563249_eng. pdf? sequence $=1$ \&isAllowed $=y$

5. Ministerio de Salud de Chile. Superintendencia de Salud. Observatorio de Calidad en Salud Guía Práctica Calidad y Seguridad Asistencial en Chile para alumnos de carreras de la salud [Internet]. Santiago de Chile: Unidad de Asesoría Técnica Intendencia de Prestadores; Feb 2018 [citado 16 
may 2021]. Disponible en: https://supersalud.gob. cl/observatorio/671/articles-16609_recurso_1.pdf

6. Rangel-Carreño TL, Lugo-Garzon IK, Calderón ME. Revisión Bibliográfica de equipos de trabajo: enfoque cuantitativo, características e identificación de variables que afectan la eficiencia. Rev Ingeniería Solidaria [Internet]. 2018 Ene [citado 14 jul 2021]; 14(24). Disponible en: https://doi.org/10.16925/ in.v14i 24.2164

7. Mohanty A, Mohanty S. The impact of communication and group dynamics on teamwork effectiveness: The case of service sector organisations. ASMJ [Internet]. 2018 [citado 16 jul 2021]; 17(4): 1-14. Disponible en: https://www.abacademies. org/articles/The-impact-of-communication-andgroup-dynamics-1939-6104-17-4-251.pdf

8. Cervantes G, Muñoz G, Inda AD. El trabajo en equipo y su efecto en la calidad del servicio a clientes. Revista Espacios [Internet]. 2020 [citado 16 jul 2021];41(14). Disponible en: http://www. revistaespacios.com/a20v41n14/a20v41n14p27. pdf

9. Hanefeld J, Powell-Jackson T, Balabanova D. Understanding and measuring quality of care: dealing with complexity. Bull World Health Organ [Internet]. 2017 May [citado 13 jul 2021]; 95(5): 368-374. Disponible en: https://doi.org/10.2471/ BLT.16.179309

10. Rosen MA, Diaz Granados D, Dietz AS, Benishek LE, Thompson D, Pronovost PJ, Weaver SJ. Teamwork in healthcare: Key discoveries enabling safer, high-quality care. Am Psychol [Internet]. May-Jun 2018 [citado 13 jul 2021]; 73(4): 433450. Disponible en: https://doi.org/10.1037/amp 0000298

11. Chassin MR. Improving the quality of health care: what's taking so long? Health Aff (Millwood) [Internet]. 2013 Oct [citado 12 jul 2021]; 32(10): 1761-5. Disponible en: https://doi.org/10.1377/ hlthaff.2013.0809

12. National Academies of Sciences, Engineering, and Medicine; Health and Medicine Division; Board on Health Care Services; Board on Global Health; Committee on Improving the Quality of Health Care Globally. Crossing the Global Quality Chasm: Improving Health Care Worldwide [Internet]. Washington (DC): National Academies Press (US); 2018 Aug [citado 16 jul 2021]. Disponible en: https://www.ncbi.nlm.nih.gov/books/NBK5356 53/ doi: 10.17226/25152

13. Weller J, Boyd M, Cumin D. Teams, tribes and patient safety: overcoming barriers to effective teamwork in healthcare. Postgrad Med J [Internet]. 2014 [citado 12 jul 2021]; 90(1061): 149-54. Disponible en: http://dx.doi.org/10.1136/postgrad medj-2012-131168

14. Heifetz R, Grashow A, Linsky M. Diagnosing the adaptive challenge: understanding human dimensions of change. In: The Practice of Adaptive Leadership: Tools and Tactics to Change Your Organization and the World. Harvard Business Review [Internet]. 2009 [citado 16 jul 2021]. Disponible en: https://hbsp.harvard.edu/product/ 3276BC-PDF-ENG

15. Leonard M, Graham S, Bonacum D. The human factor: the critical importance of effective teamwork and communication in providing safe care. Qual Saf Health Care [Internet]. 2004 [citado 13 jul 2021]; 13(Suppl 1): i85-90. Disponible en: https:// doi.org/10.1136/qhc.13.suppl_1.i85

16. Kossaify A, Hleihel W, Lahoud JC. Team-based efforts to improve quality of care, the fundamental role of ethics, and the responsibility of health managers: monitoring and management strategies to enhance teamwork. Public Health [Internet]. 2017 [citado 13 jul 2021]; 153: 91-98 Disponible en: https://doi.org/10.1016/j.puhe.2017.08.007

17. Hilton K, Anderson A. IHI Psychology of Change Framework to Advance and Sustain Improvement. IHI White Paper. Boston, Massachusetts: Institute for Healthcare Improvement; 2018 [citado 07 jul 2021]. Disponible en: https:// qi.elft.nhs.uk/wp-content/uploads/2019/10/ IHIPsychologyofChangeFrameworkWhitePaper-1. pdf

18. Herkes J, Churruca K, Ellis LA, Pomare C. Braithwaite J. How people fit in at work: systematic review of the association between personorganisation and person-group fit with staff outcomes in healthcare. BMJ [Internet]. 2019 [citado 05 oct 2021]; 9(5): e026266. Disponible en: https://doi.org/10.1136/bmjopen-2018-026266

19. Walton RE. From control to commitment in the workplace. Harv Bus Rev [Internet]. 1985 [citado 16 jul 2021]. Disponible en: https://hbr. org/1985/03/from-control-to-commitment-in-theworkplace

20. Báez ML, Cadoche L. Habilidades de Dirección en las organizaciones. Madrid, Euderma. 95 p. En: Báez ML, Cadoche L. Habilidades sociales en grupos de estudio en la Facultad de Ciencias Veterinarias de la Universidad Nacional del Litoral (Esperanza Santa Fe, Argentina) Rev Inv Vet Perú [Internet]. 2019 [citado 13 jul 2021]; 30(4): 1790-1795. Disponible en: https://doi.org/10.15381/rivep.v30i4.17266

21. Oficina de las Naciones Unidas contra la droga y el delito. Serie de módulos universitarios. Integridad y Ética. Módulo 14. Ética profesional [Internet]. Viena: Naciones Unidas; 2019 [citado 16 may 2021]; 30. Disponible en: https://www.unodc.org/ 
e4j/es/integrity-ethics/module-14/index.html

22. Rapimán, ME, Acevedo I, Osorio M, Torres A. Paul Ricoeur y una vida buena con otros y para otros: a propósito del trabajo en equipo en salud. Acta Bioeth [Internet]. 2020 [citado 13 jul 2021]; 26(1): 37-42. Disponible en: http://dx.doi.org/10.4067/ S1726-569X2020000100037

23. Lupano ML, Castro A. Influencia de virtudes organizacionales sobre satisfacción, compromiso y performance laboral en organizaciones argentinas. Interdisciplinaria [Internet]. 2019 Jun [citado 16 may 2021]; 35(1): 171-188. Disponible en: https:// www.redalyc.org/journal/180/18058784010/html/

24. Gastelu E, Arce P. Compromiso organizacional y contrato psicológico en el personal de ventas de una organización distribuidora de productos de belleza. Ajayu [Internet]. 2021 [citado 16 may 2021]; 19(1): 20-58. Disponible en: http://www.scielo. org.bo/pdf/rap/v19n1/v19n1_a02.pdf

25. Vargas F, Machicao C. Estudio comparativo del compromiso organizacional en trabajadores con y sin hijos, dentro de una empresa industrial en la ciudad de La Paz. Fides Et Ratio [Internet]. 2020 Sep [citado 24 jun 2021]; 20(2): 17-36. Disponible en: http://www.scielo.org.bo/pdf/rfer/ v20n20/v20n20_a03.pdf

26. Vargas I, Soto S, Hernández M, Campos S. La confianza en la relación profesional de la salud y paciente. RCSP [Internet]. 2020 Sep [citado 16 jul 2021]; 46(3): e1575. Disponible en: http:// scielo.sld.cu/pdf/rcsp/v46n3/1561-3127-rcsp-4603-e1575.pdf
27. Reina D, Reina M, Hunt D. Porque la confianza es clave para el éxito del equipo. Informe de Investigación. Reina A Trust Building Consultancy y Center for Creative Leadership [Internet]. 2018 [citado 16 jul 2021]; 16 p. Disponible en: https:// www.ccl.org/wp-content/uploads/2018/01/ WP-Why-Trust-Is-Critical-to-Team-Success-ESJan-2018.pdf

28. Febré N, Mondaca-Gómez K, Méndez-Celis $P$, Badilla-Morales V, Soto-Parada P, Ivanovic P, et al. Calidad en enfermería: su gestión, implementación y medición. RMCLC [Internet]. 2018 [citado 3 oct 2021]; 29: 278-87. Disponible en: https://doi. org/10.1016/j.rmclc.2018.04.008

29. Gillet N, Fouquereau E, Coillot H, Cougot B, Moret $L$, Dupont $S$, et al. The effects of work factors on nurses' job satisfaction, quality of care and turnover intentions in oncology. J Adv Nurs [Internet]. 2018 May [citado 3 oct 2021]; 74(5): 1208-1219. Disponible en: https://doi.org/10.1111/jan.13524

30. Rydenfält C, Odenrick P, Larsson PA. Organizing for teamwork in healthcare: an alternative to team training? J Health Organ Manag [Internet]. 2017 [citado 13 jul 2021]; 31(3): 347-362. Disponible en: https://doi.org/10.1108/JHOM-12-2016-0233

31. Braithwaite J, Hermes J, Ludlow K, Testa L, Lamprell G. Association between organisational and workplace cultures, and patient outcomes: systematic review. BMJ Open [Internet]. 2017 [citado 13 jul 2021]; 7(11): e017708. Disponible en: https://doi.org/10.1136/bmjopen-2017-017708 